\title{
miR-130b regulates the proliferation, invasion and apoptosis of glioma cells via targeting of CYLD
}

\author{
ZHI-QIANG XIAO $^{1^{*}}$, TENG-KUN YIN ${ }^{2 *}$, YA-XING LI ${ }^{3 *}$, JIAN-HE ZHANG ${ }^{2}$ and JIAN-JUN GU ${ }^{4,5}$ \\ ${ }^{1}$ Department of Neurosurgery, The First People's Hospital of Shangqiu, Shangqiu, Henan 476100; \\ ${ }^{2}$ Department of Neurosurgery, Fuzong Clinical College, Fujian Medical University, Fuzhou, Fujian 350025; \\ ${ }^{3}$ Department of Oncology, Taizhou People's Hospital Affiliated to the Medical College of Nantong University, \\ Taizhou, Jiangsu 225300; ${ }^{4}$ Stroke Center, Zhengzhou University People's Hospital, Henan Provincial People's Hospital, \\ Zhengzhou, Henan 450000; ${ }^{5}$ Department of Neurosurgery, Fuzhou General Hospital, Fuzhou, Fujian 350025, P.R. China
}

Received October 14, 2016; Accepted November 15, 2016

DOI: $10.3892 /$ or.2017.5651

\begin{abstract}
MicroRNAs are short non-coding RNAs that play important roles in gliomas. However, the role of miR-130b in glioma remains unclear. In the present study, miR-130b expression was upregulated in glioma tissues and cell lines. Kaplan-Meier analysis indicated that the upregulation of miR-130b expression correlated with poor prognoses in glioma patients. Multivariate analysis demonstrated that this upregulation and a high-grade classification were independent factors that both predicted poor outcomes for glioma patients. Dual-luciferase assays identified that the cylindromatosis (CYLD) gene is a direct target of miR-130b. Functional studies demonstrated that a miR-130b mimic significantly promoted the growth and invasion of glioma cells, while also inhibiting apoptosis via selective targeting of CYLD, which was enhanced by CYLD-targeted siRNA. In contrast, a miR-130b inhibitor suppressed these biological behaviors, and this inhibition was reversed by CYLD-targeted siRNA. These data revealed that miR-130b could act as a novel potential diagnostic biomarker for glioma, while also demonstrating the importance of miR-130b in the cell proliferation and progression of glioma, indicating that it may serve as a useful therapeutic target for glioma.
\end{abstract}

\section{Introduction}

Glioma is the most common malignant tumor among primary tumors of the central nervous system in adults and is divided

Correspondence to: Dr Jian-Jun Gu, Stroke Center, Zhengzhou University People's Hospital, Henan Provincial People's Hospital, Zhengzhou, Henan 450000, P.R. China

E-mail: gujianjundt@163.com

*Contributed equally

Key words: glioma, miR-130b, cylindromatosis, proliferation, invasion, apoptosis, prognosis into 4 pathological grades (I-IV) based on the classification criteria published by the World Health Organization (WHO). The most malignant type of glioma is glioblastoma (1). Although glioma can be treated by comprehensive regimens, including postoperative radiotherapy in combination with temozolomide chemotherapy, excessive glioma cell proliferation, apoptotic resistance, advanced invasion and infiltration, and chemotherapeutic drug resistance can result in unsatisfactory treatment outcomes. Patients with diffused astrocytoma have the best prognosis, with a median postoperative survival of 6-8 years, while glioblastoma patients have the poorest prognosis (median survival of 12-15 months) $(2,3)$. Hence, identification of molecular targets that can effectively suppress the malignant activity of glioma cells or be used to determine glioma development and prognosis may have important clinical value for treating glioma.

MicroRNAs (miRNAs) are endogenous small ( 22 nucleotide) non-coding RNAs that regulate post-transcriptional gene expression primarily through full or partial integration with the 3'-untranslated region (3'-UTR) of target mRNAs (4). Recent studies have revealed a number of miRNAs that are involved in the incidence, development, metastasis and drug resistance of glioma. High or low expression of miRNAs plays similar roles to oncogenes or tumor suppressor genes, which are often associated with glioma staging, thus demonstrating the potential for miRNAs to act as therapeutic targets (5-8). The miR-130 family includes miR-130a and miR-130b, which are located in the 22q11 locus. Recently, miR-130b was demonstrated to have tumor suppressive and cancer-promoting properties depending on the tumor type. miR-130b is highly expressed in melanoma (9), gastric (10), bladder (11) and colorectal cancer (12), esophageal squamous cell carcinoma (13) and glioma (14), but significantly and poorly expressed in papillary thyroid carcinomas (15), endometrial cancer (16), pituitary adenomas (17) and pancreatic cancer (18). Previously, miR-130b was observed to be overexpressed in glioma tissues, while the suppression of the expression of miR-130b via peroxisome proliferator-activated receptor- $\gamma$ (PPAR- $\gamma$ ) inhibited the proliferation and invasive activity of glioma (14).

The present study utilized quantitative PCR to detect miR-130b expression in 129 glioma specimens, assessing the 
correlation between miR-130b expression and the clinicopathological characteristics of glioma patients. In addition, both the Kaplan-Meier method and Cox's proportional hazard model were used to analyze the prognostic potential of miR-130b expression in glioma. Target gene prediction software was used to predict a potential target gene of miR-130b, cylindromatosis $(C Y L D)$ gene, this was followed by the construction of a wild-type (WT) $C Y L D$ gene and a dual-luciferase reporter plasmid with a mutant 3'-UTR sequence. A Dual-Luciferase Reporter Assay System was used to determine the effect of miR-130b on CYLD expression. The role of CYLD in cell proliferation, the cell cycle, cell migration and cell invasion were investigated using a miR-130b mimic and inhibitor in U87 and U251 cells, and by upregulating and downregulating the expression of miR-130b, as well as by utilizing a CYLDtargeted siRNA.

\section{Materials and methods}

Clinical samples. Glioma specimens from 129 glioma patients and normal brain tissues from 20 patients with severe traumatic brain injury and craniotomy were collected from the Department of Neurosurgery at Fuzhou General Hospital (Fuzhou, China) from January 2006 to December 2010. The research protocol was approved by the Ethics Committee of Fuzhou General Hospital, and all patients or their families signed informed consent forms before participating in the study. Pathological and diagnostic results of the patients were assessed and confirmed by two experienced neuropathologists. The glioma malignancy of the patients was categorized according to the classification criteria published by the WHO in 2007, including 45 cases of grades I/II and 84 cases of grades III/IV gliomas. None of the patients had undergone preoperative chemotherapy or radiotherapy. All tissue specimens were rapidly frozen in liquid nitrogen after resection for subsequent analysis. Follow-up with the patients was terminated in December 2015.

RNA extraction and quantitative real-time PCR (RT-PCR). TRIzol reagent was used to extract total RNA from glioma tissues and cells, as described in the manufacturer's instructions. miScript Reverse Transcription kit (Qiagen) was used for cDNA synthesis, followed by quantitative RT-PCR using the PCR reaction mixture ( $20 \mu 1 /$ reaction) recommended by the miScript SYBR-Green PCR kit instructions. After reverse transcription, primers specific to miR-130b, or an internal reference (U6) were used in the PCR system. A Prism 7500 RT-PCR system was used to perform qRT-PCR using the following amplification conditions: $95^{\circ} \mathrm{C}$ denaturation for $10 \mathrm{sec} ; 40$ cycles of $95^{\circ} \mathrm{C}$ denaturation for $5 \mathrm{sec}$ and $60^{\circ} \mathrm{C}$ annealing for $34 \mathrm{sec}$. The experiment was repeated 3 times. The $2^{-\Delta \Delta \mathrm{Ct}}$ method was used to calculate the relative expression of the target gene (miR-130b) based on the internal reference gene expression.

Cell culture and transfection. Glioma cell lines were purchased from the Shanghai Institute of Biochemistry and Cell Biology (Shanghai, China). Glioma cells were cultured in Dulbecco's modified Eagle's medium (DMEM) (containing $10 \%$ fetal bovine serum), and incubated at $37^{\circ} \mathrm{C}$ in a humid atmosphere with $5 \% \mathrm{CO}_{2}$ humidity. Conventional replacement of culture medium was performed when cells reached logarithmic growth and a $90 \%$ fusion rate, to continue culturing cells. Twenty-four hours before transfection, $1 \times 10^{5}$ glioma U87 and U251 cells under optimized growth conditions were independently seeded into the wells of a 6-well culture plate. Upon reaching $80 \%$ confluency, transfection was performed using Lipofectamine $^{\mathrm{TM}} 2000$ as described in the manufacturer's instructions to transfect NC, miR-130b mimic, miR-130b inhibitor, and CYLD-targeted siRNA at a $100 \mathrm{nM}$ final concentration (Shanghai GenePharma Co., Ltd., Shanghai, China) in U87 and U251 cells. Standard culture medium was replaced to continue culturing the cells $6 \mathrm{~h}$ after transfection. The transfection was repeated in triplicate.

Luciferase reporter assay. The TargetScan database was used to predict miRNA/target interaction sites between $m i R-130 b$ and $C Y L D$. GenBank was used to determine the 3'-UTR sequence of the CYLD gene. The CYLD-WT 3'-UTR and $C Y L D$-mutant 3'-UTR genes were synthesized at Sangon Biotech Co., Ltd. (Shanghai, China) with NotI and XhoI restriction sites at the two ends. The genes were subsequently inserted into the multiple cloning sites of psi-CHECK2, a dual-luciferase reporter plasmid, to prepare WT and mutant clones, followed by sequencing to confirm gene identity. HEK293A cells were seeded into the wells of a 24-well plate. Each well contained $8 \times 10^{4}$ cells which were ready for transfection once the cell fusion rate reached $80 \%$. Based on the instructions for Lipofectamine $^{\mathrm{TM}} 2000,0.2 \mu \mathrm{g}$ of psi-CHECK2 and $100 \mathrm{nM}$ miR-130b inhibitor or negative control were co-transfected with HEK293A cells. Five hours after transfection, complete DMEM was used to replace the cell culture media. After culturing for $48 \mathrm{~h}$, the HEK293A cells were lysed according to the manufacturer's (Promega, Madison, WI, USA) instructions and labeled with the luciferase substrate to detect the relative activity of luciferase in the cells.

Cell Counting Kit-8 (CCK-8). After trypsinization, $\sim 5 \times 10^{3}$ cells were seeded into the wells of a 96-well plate. Each sample was seeded into 5-wells, followed by the addition of cell culture medium and CCK-8 working solution. Blank controls contained only culture medium and CCK- 8 working solution. The optical densities (ODs) of each group of cells were assessed at $450 \mathrm{~nm}$ using a microplate reader at 24, 48, 72 and $96 \mathrm{~h}$ after transfection. The following formula was used to calculate growth inhibition and to prepare a growth inhibition curve: Cell growth inhibition $=(1-$ OD of the experimental group/OD of the control group) $x 100 \%$. This experiment was repeated 3 times.

Cell cycle assay. Freshly-prepared $70 \%$ pre-chilled ethanol was used for fixing cells and permeabilization occurred at $4^{\circ} \mathrm{C}$ overnight. Each group of cells was digested and mixed to form a single cell suspension. The collected cells were washed once in phosphate-buffered saline (PBS) and fixed with 70\% pre-chilled ethanol at $4^{\circ} \mathrm{C}$ overnight. After being washed once with PBS, the cell suspension was filtered through a 400-mesh filter. RNase A was incubated with the cell suspension for $30 \mathrm{~min}$, followed by the staining of the cells with propidium iodide (PI) for $30 \mathrm{~min}$ in the dark. The cell cycle distribution 
was assessed by flow cytometry and the experiment was repeated in triplicate.

\section{Invasion and migration assays}

Invasion assay. Matrigel matrix was dissolved at $4^{\circ} \mathrm{C}$ overnight, then, diluted at a 1:3 ratio with pre-chilled serum-free medium by adding $40 \mu \mathrm{l}$ of the diluent into a pre-chilled Transwell culture plate, followed by the covering of the plate with a polycarbonate resin film and incubation at $37^{\circ} \mathrm{C}$ for $2 \mathrm{~h}$ to solidify the Matrigel matrix. A $100 \mu$ l cell suspension $\left(5 \times 10^{4}\right.$ cells) was added into the Transwell culture plate, followed by $200 \mu \mathrm{l}$ of serum-free medium. Chemokine $(500 \mu \mathrm{l})$ was added to the lower chamber of the Transwell culture plate, and the cells were incubated at $37^{\circ} \mathrm{C}$ for $24 \mathrm{~h}$ in the incubator containing $5 \%$ $\mathrm{CO}_{2}$. The upper Transwell chamber was removed in such a way so that the culture medium could be aspirated from inside the chamber. The residual cells and Matrigel matrix were wiped onto the upper Transwell chamber. The culture medium of the lower Transwell chamber was aspirated, followed by immerse washing of the chamber twice with PBS. Paraformaldehyde (4\%) was used to fix the chamber membrane for $15 \mathrm{~min}$, and then the chamber was washed twice with PBS and stained with $0.1 \%$ crystal violet solution at room temperature for $15 \mathrm{~min}$. After being washed 3 times with PBS, the membrane of each small chamber was collected and mounted onto a glass slide for imaging under microscopy.

Migration assay. For migration assays, the Transwell upper chamber was not coated with Matrigel matrix. The remaining procedures were similar to the invasion assay. Both the invasion and migration assays were repeated 3 times.

Tumor inoculation in nude mice. Pre-transfected cells in each group were prepared as previously described. After transfection of the cells for $48 \mathrm{~h}$, the cell suspension in each group was prepared to adjust the single cell suspension to $4 \times 10^{6}$ cells $/ 200 \mu 1$. The left armpit of each nude mouse was used as a culturing site for the disinfection with ethanol and the subcutaneous injection of the $200 \mu \mathrm{l}$ single cell suspension, followed by acupressure to avoid overflow of the cell suspension from the culturing site. A Vernier caliper was used to assess and record the tumor diameter (a) and width (b) every 7 days for 42 days. Each nude mouse was euthanized via decapitation to assess the tumor size, after which the tumor was completely resected and the tumor was preserved at $-80^{\circ} \mathrm{C}$ for later use in the immunoblot analysis. The tumor volume (V) was calculated according to the following equation: $\mathrm{V}=\left(a b^{2}\right) / 2$. The animal studies were approved by the Institutional Animal Care and Use Committee of the Fuzhou General Hospital, Fuzhou, China.

Immunoblot analysis. U87 and U251 glioma cells were collected $48 \mathrm{~h}$ after transfection and added to RIPA protein lysate for total protein extraction, which was quantified via BCA assay. Total protein $(40 \mu \mathrm{g})$ extracted from each culture were separated using SDS-PAGE and transferred onto a polyvinylidene fluoride (PVDF) membrane. Each blot was blocked with $5 \%$ skim milk at room temperature for $1 \mathrm{~h}$, followed by incubation with anti-CYLD monoclonal antibody $(1: 1,000)$ at $4^{\circ} \mathrm{C}$ overnight. After washing with Tris-buffered saline with
Table I. Relationship between miR-130b expression and clinicopathological features.

\begin{tabular}{|c|c|c|c|c|}
\hline \multirow[b]{2}{*}{$\begin{array}{l}\text { Clinicopathological } \\
\text { features }\end{array}$} & \multirow[b]{2}{*}{ Pts. } & \multicolumn{2}{|c|}{$\begin{array}{c}\text { miR-130b } \\
\text { expression } \\
\text { median value }\end{array}$} & \multirow[b]{2}{*}{ P-value } \\
\hline & & $\begin{array}{l}\text { Low } \\
\text { (n) }\end{array}$ & $\begin{array}{l}\text { High } \\
\text { (n) }\end{array}$ & \\
\hline Age (years) & & & & 0.193 \\
\hline$<50$ & 50 & 17 & 33 & \\
\hline$\geq 50$ & 79 & 36 & 43 & \\
\hline Sex & & & & 0.307 \\
\hline Male & 75 & 25 & 29 & \\
\hline Female & 54 & 28 & 47 & \\
\hline WHO grade & & & & $0.000^{\mathrm{a}}$ \\
\hline Low & 45 & 36 & 9 & \\
\hline High & 84 & 17 & 67 & \\
\hline KPS score & & & & $0.010^{\mathrm{a}}$ \\
\hline$<90$ & 61 & 43 & 38 & \\
\hline$\geq 90$ & 68 & 10 & 28 & \\
\hline
\end{tabular}

${ }^{\text {a }} \mathrm{P}<0.05$. Pts., patients; KPS, Karnofsky performance status; WHO, World Health Organization; Low, WHO I/II; High, WHO III/III.

Tween-20 (TBST) the blot was incubated with horseradish peroxidase-labeled secondary antibody $(1: 2,000)$ at room temperature for $1 \mathrm{~h}$. Finally, the blot was washed with TBST washing, developed with enhanced chemiluminescence (ECL) reagent, and photographed to visualize CYLD on the blot. The relative protein content of CYLD was assessed based on the gray scale value of the protein band.

Statistical analysis. The SPSS 19.0 software (SPSS, Inc., Chicago, IL, USA) was used for statistical analysis. All data are presented using the mean \pm standard deviation. ANOVA was used for the comparison between groups. The KaplanMeier method and log-rank test were used to calculate and compare patient survival, respectively. Multivariate Cox regression analysis was used to assess predictors related to survival. $\mathrm{P}<0.05$ was considered to be statistically significant. All experiments were independently repeated in triplicate.

\section{Results}

miR-130b upregulation is associated with unfavorable clinicopathological parameters in human glioma. The relative miR-130b expression was assessed by RT-PCR using miRNAs isolated from 129 glioma specimens, 26 normal brain tissues, and the U87 and U251 glioma cell lines. The relative miR-130b expression in glioma tissue and cell lines was significantly higher than in normal brain tissues and primary normal human astrocytes (NHA; $\mathrm{P}<0.05$; Fig. 1A and $\mathrm{B}$ ), suggesting that miR-130b may play a role as an oncogene in glioma. A correlation analysis between miR-130 expression and clinicopathological features/glioma prognosis indicated that 
A

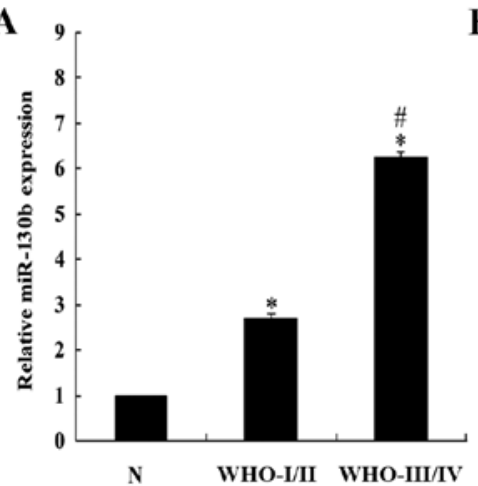

B

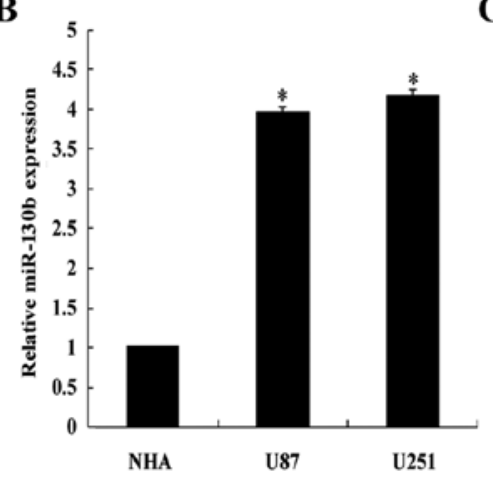

C

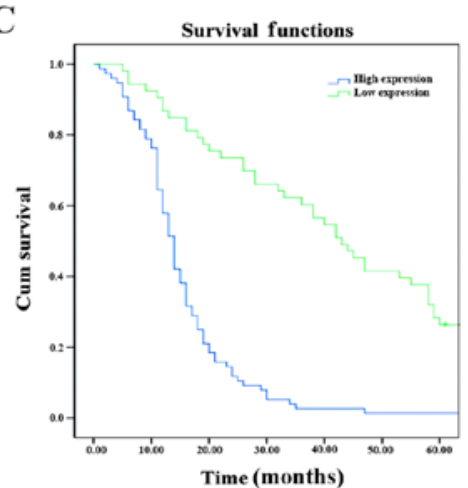

D

Position 190-196 of CYLD 3'-UTR 5'...UUAAUAAGAAGCAUUUUGCACUC 3'

|11111

miR-130b 3' UACGGGAAAGUAGUAACGUGAC 5 ,

$\mathbf{E}$

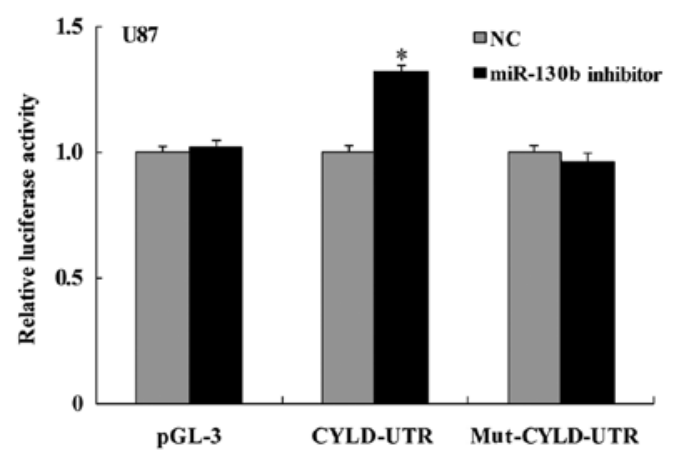

F

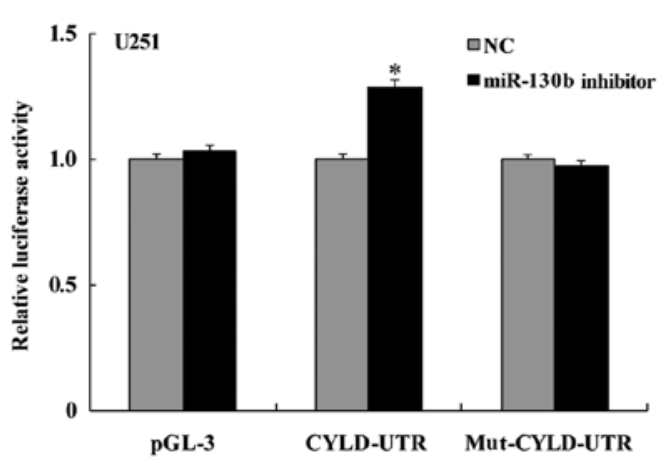

Figure 1. (A) Real-time PCR analysis of miR-130b expression in glioma tissues; ${ }^{*} \mathrm{P}<0.05$ vs. the non-neoplastic brain specimens; ${ }^{*} \mathrm{P}<0.05$ vs. the WHO I/II groups. (B) Real-time PCR analysis of miR-130b expression in glioma U87 and U251 cells, and primary normal human astrocytes (NHA); ${ }^{*}<0.05$ vs. the NHA cells. (C) Kaplan-Meier curves of the survival time in patients with glioma divided according to miR-130b expression (P<0.05). (D) Identification of miR-130b target sites in the 3'-UTR of CYLD performed on TargetScan. (E and F) Luciferase assay; ${ }^{*} \mathrm{P}<0.05$.

miR-130 expression was not associated with the sex and age of glioma patients $(\mathrm{P}>0.05)$, but it was significantly associated with the WHO glioma classification grading scale and the Karnofsky performance status (KPS) scale ( $<<0.05$; Table I). The median survival of patients with upregulated miR-130b expression was significantly lower than patients with low miR-130b expression ( $14 \pm 0.717$ and $43 \pm 4.094$, respectively; $\mathrm{P}<0.05)$. The 5-year survival of patients with upregulated miR-130b expression was significantly lower than patients with low miR-130b expression $(\mathrm{P}<0.001$; Fig. 1C). Multivariate Cox's model analysis indicated a high pathological grade for glioma $(\mathrm{P}=0.002)$ and miR-130b overexpression $(\mathrm{P}=0.000)$, which are two independent factors used to predict the survival of glioma patients (Table II).

CYLD is a potential downstream target of miR-130b. Using a prediction algorithm through the TargetScan bioinformatics database, the 3'-UTR of CYLD contained several miR-130b interaction sites, including a seed sequence. This seed sequence is located at the 190-196th nucleotides of the phosphatase and tensin homolog 3'-UTR (Fig. 1D). A reporter construct was designed by ligating the 3'-UTR of the CYLD fragment (containing the predicted miR-130b interaction sites) downstream of a luciferase reporter gene. Transfection of the construct demonstrated a significant increase $(\mathrm{P}<0.05)$ in lucif-
Table II. Multivariate analyses of prognostic parameters in the glioma patients using Cox regression analysis.

\begin{tabular}{lccc}
\hline & \multicolumn{3}{c}{ Multivariable analysis } \\
\cline { 2 - 4 } Variable & HR & $95 \%$ CI & P-value \\
\hline Age (years) & 1.547 & $0.982-2.276$ & 0.097 \\
Sex & 1.074 & $0.738-1.564$ & 0.71 \\
WHO grade & 2.992 & $1.509-5.931$ & $0.002^{\mathrm{a}}$ \\
KPS score & 1.602 & $0.903-2.841$ & 0.107 \\
miR-130b & 2.910 & $1.736-4.875$ & $0.000^{\mathrm{a}}$
\end{tabular}

WHO, World Health Organization; KPS, Karnofsky performance status; HR, hazard ratio; CI, confidence interval.

erase activity of the WT reporter plasmid after co-transfection with the miR-130b inhibitor. In contrast, the luciferase activity using the mutant plasmid was not significantly different from the control group $(\mathrm{P}>0.05$; Fig. $1 \mathrm{E}$ and $\mathrm{F})$.

miR-130b regulates the proliferation of $U 87$ and $U 251$ cells. Both CCK- 8 and colony formation assays were utilized to validate the 
A

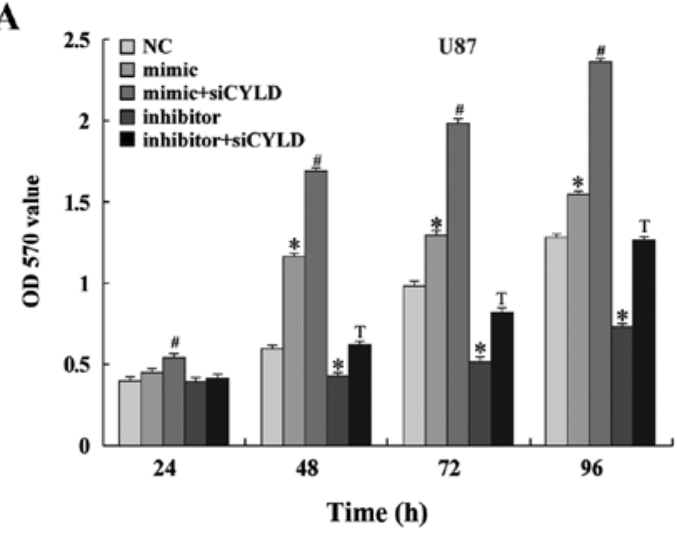

B

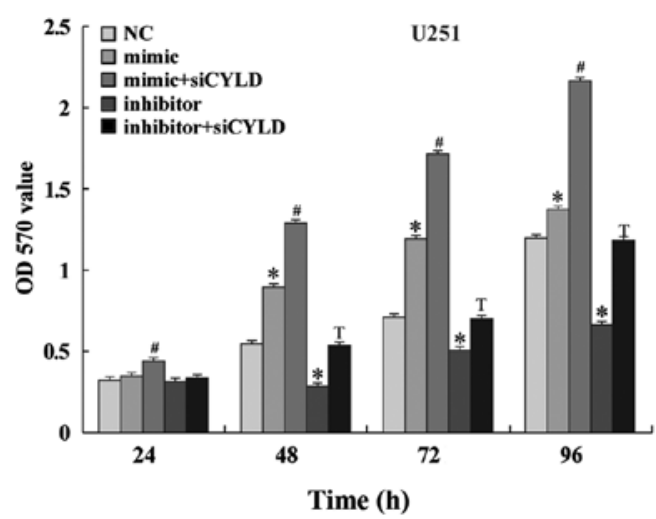

C

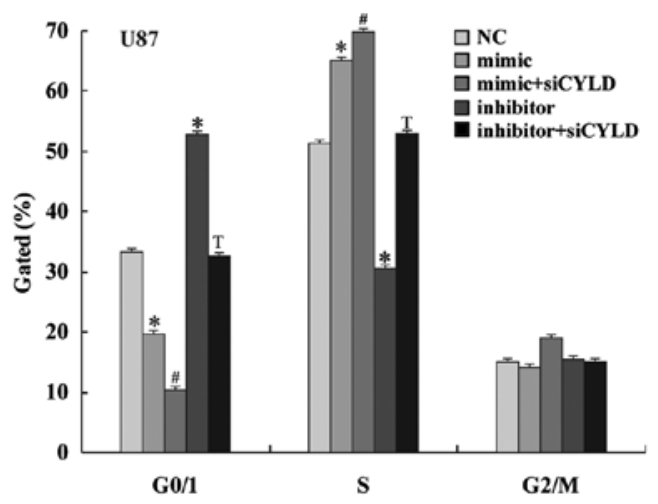

E

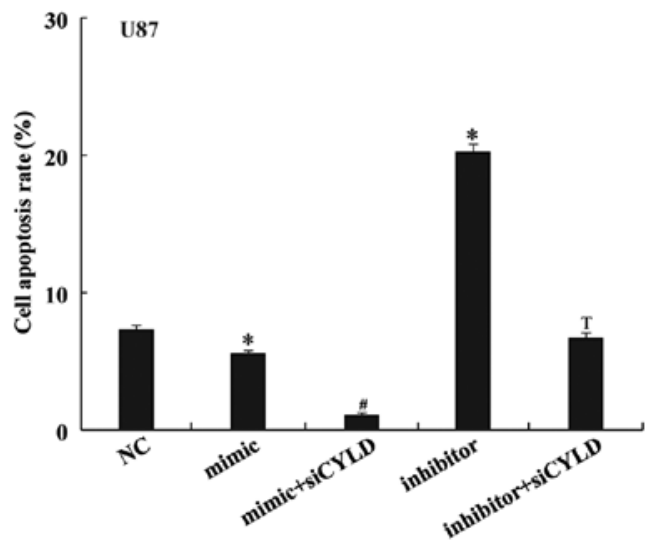

D

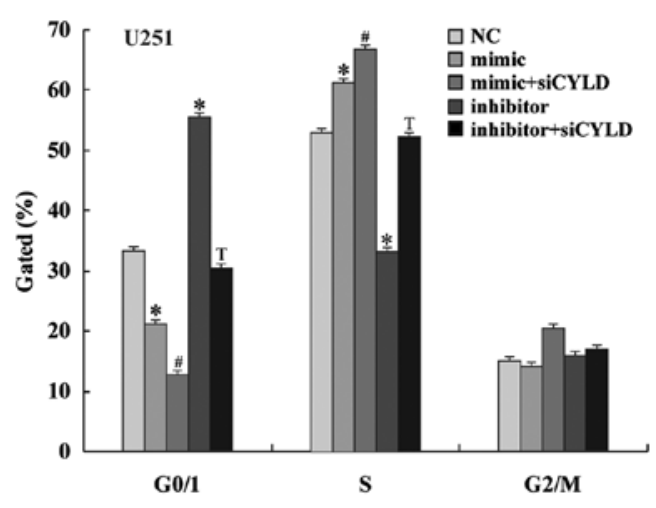

F

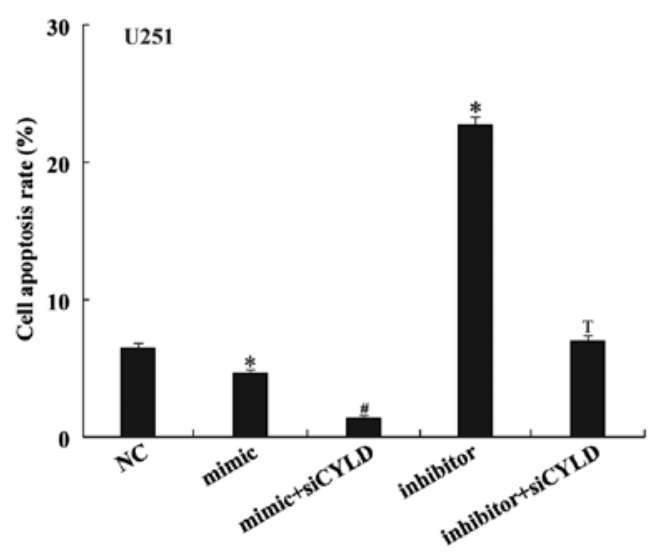

Figure 2. (A and B) CCK-8 assays revealed cell growth inhibition of indicated cells. (C and D) Flow cytometric determination of the proportion of cells in distinct cell cycle phases. (E and F) Flow cytometry revealed the proportion of apoptotic glioma cells. ${ }^{*} \mathrm{P}<0.05$ vs. the NC group; ${ }^{*} \mathrm{P}<0.05$ vs. the mimic group; ${ }^{\mathrm{T}} \mathrm{P}<0.05$ vs. the inhibitor group. Data are presented as the mean $\pm \mathrm{SD}$ of 3 independent experiments.

impact of miR-130b on cell proliferation in U87 and U251 cell lines. Twenty-four hours post-transfection, the glioma cells were collected and the cell proliferation was assessed at 24,48,72 and $96 \mathrm{~h}$ after the cells were replaced. Glioma cell proliferation was significantly enhanced at 48,72 and $96 \mathrm{~h}$ after transfection with the miR-130b mimic compared to the negative control $(\mathrm{P}<0.05$; Fig. 2A and B). Additionally, $24 \mathrm{~h}$ after co-transfection with CYLD siRNA, the miR-130b mimic-induced cell proliferation was enhanced. In contrast, cell proliferation of glioma cells was significantly suppressed at 48,72 and $96 \mathrm{~h}$ after transfection with the miR-130b inhibitor; while co-transfection with CYLD siRNA suppressed the activity of the miR-130b inhibitor at these time-points. Flow cytometric analysis indicated that glioma cell counts were decreased during the G0/G1 phase, but were increased in number in the $\mathrm{S}$ phase after transient transfection with the miR-130b mimic. Co-transfection with CYLD siRNA significantly decreased the number of cells in the G0/G1 phase, while also increasing the number of cells in the $\mathrm{S}$ phase. In contrast, the number of cells at the G0/G1 phase was arrested and the cells in the $\mathrm{S}$ phase were decreased after transfection with the miR-130b inhibitor (Fig. 2C and D); co-transfection with the miR-130b inhibitor and CYLD siRNA counteracted the effect. These in vitro experiments revealed that miR-130b affected cell proliferation by regulating the G1/S transition in the cell cycle.

miR-130b regulates apoptosis in U87 and U251 cells. In order to ascertain the effect of miR-130b on apoptosis in glioma cells, 
A

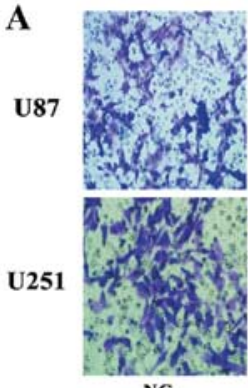

NC

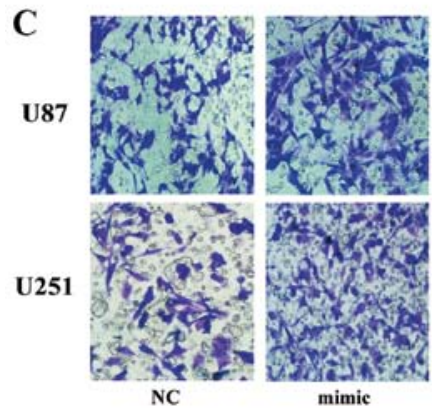

NC

$\mathbf{E}$
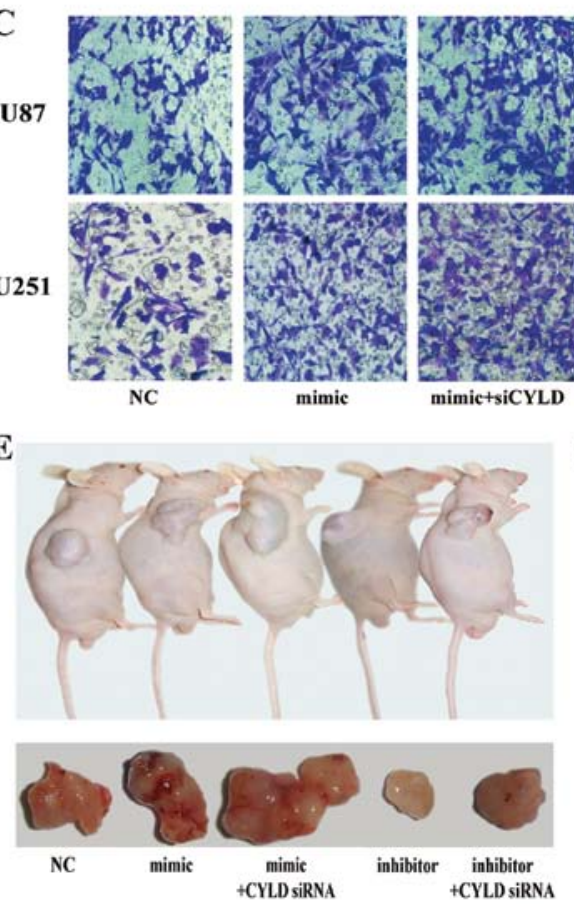
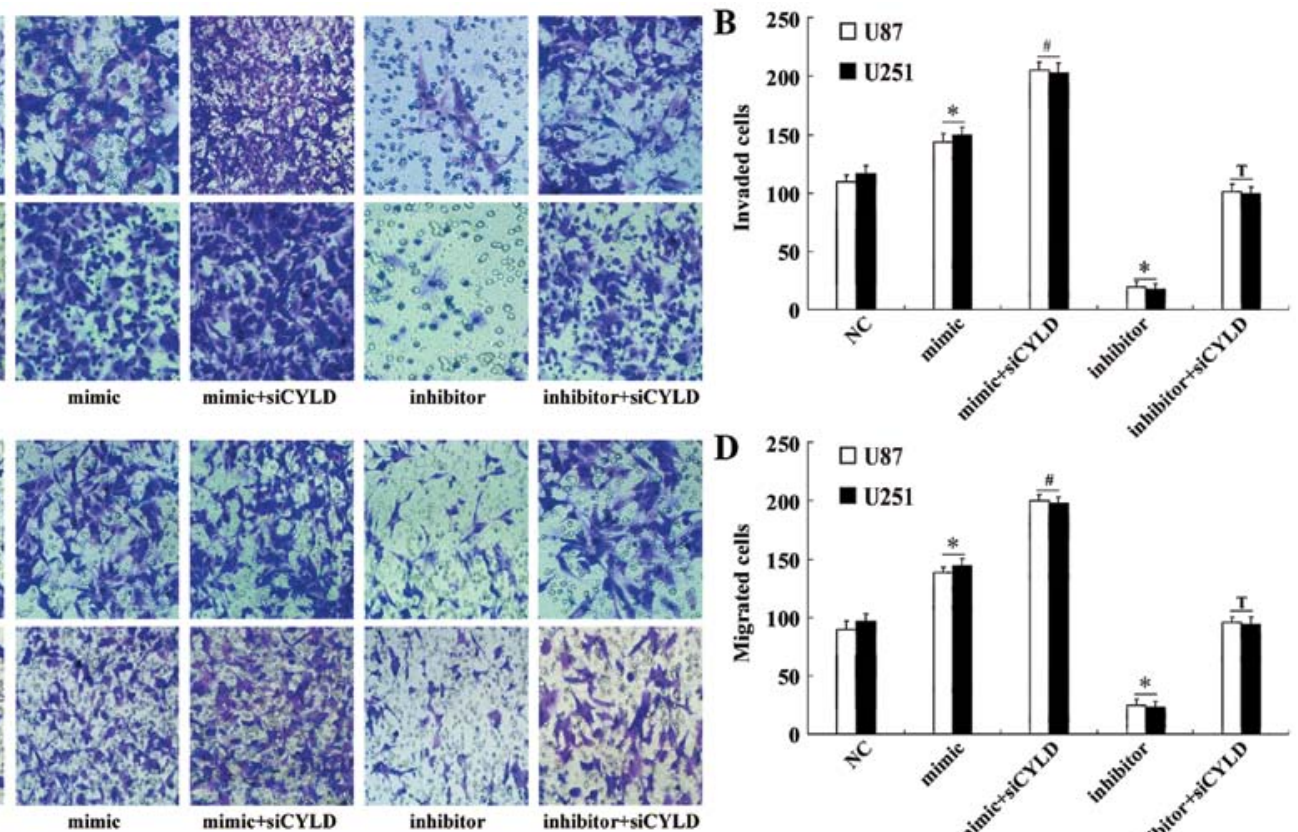

D ${ }^{250}[\square$ Q 887

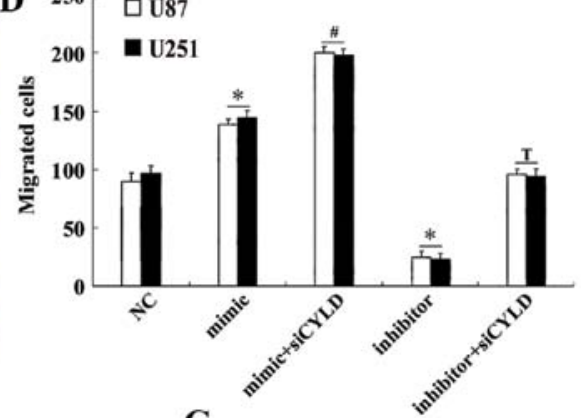

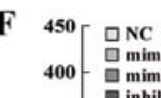

inhibitor+siCYLD

G

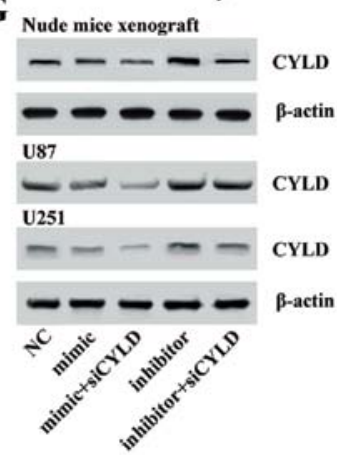

Figure 3. (A and B) Transwell assay revealed the invasive ability of transfected glioma cells. (C and D) The results of the migration experiment were consistent with the invasion experiment. (E) Representative images of tumor growth are shown. (F) The tumor volume was calculated every week after injection to determine the tumor growth. (G) The levels of CYLD protein in nude mouse xenografts and glioma cells were assessed using western blotting. ${ }^{*}<0.05$ vs. the $\mathrm{NC} ;{ }^{\text {" }} \mathrm{P}<0.05$ vs. the mimic; ${ }^{\mathrm{T}} \mathrm{P}<0.05$ vs. the inhibitor.

an Annexin V-FITC/PI double staining assay was performed on cells transfected with the miR-130b mimic. Compared to the negative control group, the number of apoptotic cells in the U87 and U251 cell lines was significantly decreased $(\mathrm{P}<0.05)$. In contrast, the number of apoptotic cells in the U87 and U251 cell lines was significantly enhanced after transfecting the cells with the miR-130b inhibitor $(\mathrm{P}<0.05)$. Co-transfection with CYLD siRNA enhanced the anti-apoptotic ability of the miR-130b mimic, while also decreasing the enhanced apoptosis of glioma cells that was induced by the miR-130b inhibitor (Fig. 2E and F).

miR-130b regulates the invasiveness and metastasis of U87 and U251 cells. Using invasion and migration assays, the impact of miR-130b on cell invasion and migration in U87 and U251 cell lines was assessed. Twenty-four hours after miR-130b mimic transfection, the amount of U87 and U251 cells that passed through the Transwell membrane was significantly higher than the negative control group $(\mathrm{P}<0.05$; Fig. $3 \mathrm{~A}$ and $\mathrm{B})$; co-transfection with siRNA further increased these invasion results. In contrast, U87 and U251 cells that were transfected with the
miR-130b inhibitor had decreased invasion in comparison to the negative control, while co-transfection with CYLD siRNA counteracted the suppressive effect of the miR-130b inhibitor on the invasiveness of glioma cells (Fig. 3A and B). The experimental results obtained with the migration assay were similar to the invasive assay results (Fig. 3C and D).

miR-130b affects glioma growth in nude mice. Nude mice were subcutaneously inoculated with in vitro-cultured U251 cells once the transfection rate reached $>90 \%$. Small tumor nodules were found at the inoculation sites of the nude mice 1-2 weeks later, with a tumor formation rate of $100 \%$, and in vivo glioma growth was monitored weekly. Tumor nodules which appeared earlier, with rapid glioma growth and large tumor volumes were found in the nude mice inoculated with glioma cells transfected with the miR-130b mimic. In contrast, tumor nodules which appeared later, with slow glioma growth and smaller tumor volumes were found in the nude mice inoculated with glioma cells transfected with the miR-130b inhibitor. Significant differences in tumor volume were observed $(\mathrm{P}<0.05$; Fig. $3 \mathrm{E}$ and $\mathrm{F})$. Immunoblot analysis 
on the glioma specimens demonstrated that CYLD expression in the glioma tissues of the miR-130b mimic group was significantly decreased, while CYLD expression in the glioma tissues of the miR-130b inhibitor group was significantly enhanced (Fig. 3G).

miR-130b regulates $C Y L D$ protein expression in $U 87$ and U251 cells. Twenty-four hours after transient transfection, changes in the expression of CYLD were assessed from total extracted protein isolated from U87 and U251 glioma cells. In comparison to the negative control group, the protein expression of CYLD was significantly decreased after the expression of miR-130b was upregulated, while the protein expression of CYLD was significantly increased after downregulation of the expression of miR-130b. The protein expression of CYLD was further decreased in glioma cells after co-transfection with CYLD siRNA and the miR-130b mimic. However, co-transfection with CYLD siRNA counteracted the effects of the miR-130b inhibitor in enhancing CYLD expression in glioma cells (Fig. 3G).

\section{Discussion}

miRNAs, a class of short non-coding small molecular RNAs which are comprised of 19-25 nucleotides, are extremely important biomolecules in the regulation of gene expression, affecting $\sim 30 \%$ of human genome transcription through full or partial integration with the 3'-UTR of target mRNAs. This results in mRNA degradation or inhibition of post-transcriptional translation, thereby altering processes related to the cell cycle, apoptosis, cell proliferation and cell migration (19). miRNA-130b is located on chromosome 22 and the mature sequence is 22 nucleotides in length. A variety of genes such as PPAR- $\gamma$ (20), STAT3 (18), PTEN (13) and Fmrl (21), that are associated with the incidence and development of cancer are also target genes for miR-130b. However, recent studies have demonstrated that miR-130b expression is either enhanced or decreased in different types of tumors, demonstrating that it serves a role as either a tumor promoter or suppressor, and may serve as a potential biomarker for tumor development and prognosis. Wu et al (22) observed upregulated miR-130b expression in clear-cell renal cell carcinoma and clinicopathological analysis indicated that miR-130b was associated with tumor metastasis. A long-term follow-up study of patients that had enhanced miR-130 expression suggested an association with poor prognoses. Zhao et al (18) observed that miR-130b expression was significantly decreased in pancreatic cancer, and a long-term follow-up study suggested that pancreatic cancer patients with low miR-130b expression had poor prognoses compared to pancreatic cancer patients with high miR-130b expression, suggesting that miR-130b shares features with tumor-suppressor genes.

In the present study, miR-130b was expressed at significantly higher levels in glioma tissue and cells in comparison to normal brain tissues and NHA. The pathological grading of glioma that had upregulated miR-130b expression was significantly higher than glioma with low miR-130 expression, suggesting that miR-130b played a similar role to cancer-promoting genes during the incidence of gliomas. A correlation analysis between clinicopathological features and prognosis of gliomas indicated that miR-130b overexpression was closely associated with a high WHO glioma grade, low KPS and short cumulative survival of glioma patients. Analysis by Cox's proportional hazard model demonstrated that the hazard ratio (HR) of glioma patients with miR-130b overexpression was 2.910-fold higher than glioma patients with low miR-130b expression. In addition, the HR of glioma patients with high pathological grading was 2.922-fold higher than glioma patients with low pathological grading, suggesting that miR-130b overexpression and high pathological grading were two independent parameters in predicting poor prognosis in glioma patients. Thus, this clinical study suggested that miR-130b could act as a marker for predicting the prognosis of glioma patients.

$C Y L D$, a tumor-suppressor gene, is extensively expressed in humans and consists of 956 amino acid residues, including a CAP-Gly and a C-terminal domain (23). Numerous studies have demonstrated that CYLD can act as a de-ubiquitination enzyme and that this activity inhibits the activation of the $\mathrm{NF}-\kappa \mathrm{B}, \mathrm{JNK}$ and $\mathrm{WNT} / \beta$-catenin signaling pathways. Thus, CYLD plays an important regulatory role in various processes, including cell cycle regulation, mediating apoptosis and inhibiting tumor incidence (24-26). Multiple studies have shown that deficiency or downregulation of $C Y L D$ is involved in the development of melanoma, $\mathrm{T}$ cell leukemia, incidence and development of colon cancer and hepatocellular carcinoma (27-29). Song et al (30) observed downregulated CYLD expression in 14 glioma tissues and 15 glioma cell lines; CYLD expression was negatively associated with the pathological WHO grading of glioma. In addition, glioma patients with low CYLD expression had shorter postoperative survival than glioma patients with higher CYLD expression. Moreover, CYLD expression was negatively associated with CD31, Ki67 and MMP-9 expression. These findings confirm that decreased CYLD expression is related to the incidence and development of glioma. Recently, an in-depth study of miRNAs in tumors revealed that CYLD is a target gene among all miRNA targets that regulate cell proliferation, apoptosis and invasion of cancers. For example, miR-454 promotes human colon cancer cell proliferation via CYLD gene expression (31); miR-182 regulates glioma cell proliferation and invasiveness by targeting the $C Y L D$ gene (30); miR-130b inhibits the proliferation and mediated apoptosis of stomach cancer cells by targeting CYLD (10).

Using the TargetScan bioinformatics database, $C Y L D$ was a predicted target gene for miR-130b. The putative mRNA 3'-UTR 'seed sequence' region of CYLD was ligated into the 3' end of a pGL-3 luciferase reporter gene, allowing the authenticity of the targets to be confirmed by co-transfection of cells with the miRNA and a modified pGL-3 luciferase reporter gene vector, simulating the miRNA regulating gene expression. This experiment confirmed that $C Y L D$ was a downstream target of miR-130b and additional in vitro experiments demonstrated that glioma cells transfected with the miR-130b mimic and inhibitor were able to suppress or promote the protein expression of $C Y L D$. Increased miR-130b expression promoted cell proliferation and cells in the G0/G1 phase and entering the $\mathrm{S}$ phase, arresting apoptosis while promoting tumor invasion and metastasis. Conversely, decreased miR-130b expression suppressed cell proliferation and cells in the G0/G1 phase and 
entering the $\mathrm{S}$ phase, induced apoptosis, and inhibited tumor invasion and metastasis. Co-transfection of the miR-130b inhibitor and CYLD siRNA reversed the inhibitory effect of the miR-130b inhibitor on tumor cell proliferation and invasiveness, while co-transfection of the miR-130 mimic and CYLD siRNA enhanced the ability of the miR-130 mimic to promote tumor cell proliferation and invasiveness. The present study demonstrated that miR-130b was associated with the activation of the CYLD signaling pathway during the incidence and development of glioma.

In conclusion, the present study confirmed that the upregulation of miR-130b expression in glioma was associated with a positive disease prognosis. miR-130b negatively regulated the expression of CYLD, a tumor-suppressor gene, in glioma and played an important role in the proliferation, apoptosis, and invasiveness of glioma cells. Therefore, miR-130b has the potential to serve as prognostic marker and therapeutic target for glioma.

\section{References}

1. Ricard D, Idbaih A, Ducray F, Lahutte M, Hoang-Xuan K and Delattre JY: Primary brain tumours in adults. Lancet 379: 1984-1996, 2012.

2. Lee JH, Jung TY, Jung S, Kim IY, Jang WY, Moon KS and Jeong EH: Performance status during and after radiotherapy plus concomitant and adjuvant temozolomide in elderly patients with glioblastoma multiforme. J Clin Neurosci 20: 503-508, 2013.

3. Wen PY and Kesari S: Malignant gliomas in adults. N Engl J Med 359: 492-507, 2008.

4. Calin GA, Liu CG, Sevignani C, Ferracin M, Felli N, Dumitru CD, Shimizu M, Cimmino A, Zupo S, Dono M, et al: MicroRNA profiling reveals distinct signatures in B cell chronic lymphocytic leukemias. Proc Natl Acad Sci USA 101: 11755-11760, 2004.

5. Weber F, Teresi RE, Broelsch CE, Frilling A and Eng C: A limited set of human MicroRNA is deregulated in follicular thyroid carcinoma. J Clin Endocrinol Metab 91: 3584-3591, 2006.

6. Jun GJ, Zhong GG and Ming ZS: miR-218 inhibits the proliferation of glioma U87 cells through the inactivation of the CDK6/cyclin D1/p21 Cip1/Waf1 pathway. Oncol Lett 9: 2743-2749, 2015.

7. Jiang L, Wang C, Lei F, Zhang L, Zhang X, Liu A, Wu G, Zhu J and Song L: miR-93 promotes cell proliferation in gliomas through activation of PI3K/Akt signaling pathway. Oncotarget 6: 8286-8299, 2015.

8. Hou SX, Ding BJ, Li HZ, Wang L, Xia F, Du F, Liu LJ, Liu YH, Liu XD, Jia JF, et al: Identification of microRNA-205 as a potential prognostic indicator for human glioma. J Clin Neurosci 20: 933-937, 2013.

9. Sand M, Skrygan M, Sand D, Georgas D, Gambichler T, Hahn SA, Altmeyer P and Bechara FG: Comparative microarray analysis of microRNA expression profiles in primary cutaneous malignant melanoma, cutaneous malignant melanoma metastases, and benign melanocytic nevi. Cell Tissue Res 351: 85-98, 2013.

10. Sun B, Li L, Ma W, Wang S and Huang C: MiR-130b inhibits proliferation and induces apoptosis of gastric cancer cells via CYLD. Tumour Biol 37: 7981-7987, 2016.

11. Egawa H, Jingushi K, Hirono T, Ueda Y, Kitae K, Nakata W, Fujita K, Uemura M, Nonomura $\mathrm{N}$ and Tsujikawa K: The miR-130 family promotes cell migration and invasion in bladder cancer through FAK and Akt phosphorylation by regulating PTEN. Sci Rep 6: 20574, 2016.

12. Colangelo T, Fucci A, Votino C, Sabatino L, Pancione M, Laudanna C, Binaschi M, Bigioni M, Maggi CA, Parente D, et al: MicroRNA-130b promotes tumor development and is associated with poor prognosis in colorectal cancer. Neoplasia 15: 1086-1099, 2013.
13. Yu T, Cao R, Li S, Fu M, Ren L, Chen W, Zhu H, Zhan Q and Shi R: MiR-130b plays an oncogenic role by repressing PTEN expression in esophageal squamous cell carcinoma cells. BMC Cancer 15: 29, 2015.

14. Gu JJ, Zhang JH, Chen HJ and Wang SS: MicroRNA-130b promotes cell proliferation and invasion by inhibiting peroxisome proliferator-activated receptor- $\gamma$ in human glioma cells. Int J Mol Med 37: 1587-1593, 2016

15. Dettmer MS, Perren A, Moch H, Komminoth P, Nikiforov YE and Nikiforova MN: MicroRNA profile of poorly differentiated thyroid carcinomas: New diagnostic and prognostic insights. J Mol Endocrinol 52: 181-189, 2014.

16. Li BL, Lu C, Lu W, Yang TT, Qu J, Hong X and Wan XP: miR-130b is an EMT-related microRNA that targets DICER1 for aggression in endometrial cancer. Med Oncol 30: 484, 2013.

17. Leone V, Langella C, D'Angelo D, Mussnich P, Wierinckx A, Terracciano L, Raverot G, Lachuer J, Rotondi S, Jaffrain-Rea ML, et al: Mir-23b and miR-130b expression is downregulated in pituitary adenomas. Mol Cell Endocrinol 390: 1-7, 2014.

18. Zhao G, Zhang JG, Shi Y, Qin Q, Liu Y, Wang B, Tian K, Deng SC, Li X, Zhu S, et al: MiR-130b is a prognostic marker and inhibits cell proliferation and invasion in pancreatic cancer through targeting STAT3. PLoS One 8: e73803, 2013.

19. Mei Q, Li F, Quan H, Liu Y and Xu H: Busulfan inhibits growth of human osteosarcoma through miR-200 family microRNAs in vitro and in vivo. Cancer Sci 105: 755-762, 2014.

20. Lee EK, Lee MJ, Abdelmohsen K, Kim W, Kim MM, Srikantan S, Martindale JL, Hutchison ER, Kim HH, Marasa BS, et al: miR-130 suppresses adipogenesis by inhibiting peroxisome proliferator-activated receptor gamma expression. Mol Cell Biol 31: 626-638, 2011.

21. Gong X, Zhang K, Wang Y, Wang J, Cui Y, Li S and Luo Y: MicroRNA-130b targets Fmrl and regulates embryonic neural progenitor cell proliferation and differentiation. Biochem Biophys Res Commun 439: 493-500, 2013.

22. Wu X, Weng L, Li X, Guo C, Pal SK, Jin JM, Li Y, Nelson RA, Mu B, Onami SH, et al: Identification of a 4-microRNA signature for clear cell renal cell carcinoma metastasis and prognosis. PLoS One 7: e35661, 2012.

23. Trompouki E, Hatzivassiliou E, Tsichritzis T, Farmer H, Ashworth A and Mosialos G: CYLD is a deubiquitinating enzyme that negatively regulates NF-kappaB activation by TNFR family members. Nature 424: 793-796, 2003.

24. Sun SC: CYLD: A tumor suppressor deubiquitinase regulating NF-kappaB activation and diverse biological processes. Cell Death Differ 17: 25-34, 2010.

25. Xue L, Igaki T, Kuranaga E, Kanda H, Miura M and Xu T: Tumor suppressor CYLD regulates JNK-induced cell death in Drosophila. Dev Cell 13: 446-454, 2007.

26. Tauriello DV, Haegebarth A, Kuper I, Edelmann MJ, Henraat M, Canninga-van Dijk MR, Kessler BM, Clevers H and Maurice MM: Loss of the tumor suppressor CYLD enhances Wnt/beta-catenin signaling through K63-linked ubiquitination of Dvl. Mol Cell 37: 607-619, 2010.

27. Massoumi R, Kuphal S, Hellerbrand C, Haas B, Wild P, Spruss T, Pfeifer A, Fässler R and Bosserhoff AK: Down-regulation of CYLD expression by Snail promotes tumor progression in malignant melanoma. J Exp Med 206: 221-232, 2009.

28. Espinosa L, Cathelin S, D'Altri T, Trimarchi T, Statnikov A, Guiu J, Rodilla V, Inglés-Esteve J, Nomdedeu J, Bellosillo B, et al: The Notch/Hes1 pathway sustains $\mathrm{NF}-\kappa \mathrm{B}$ activation through $C Y L D$ repression in T cell leukemia. Cancer Cell 18: 268-281, 2010.

29. Hellerbrand C, Bumes E, Bataille F, Dietmaier W, Massoumi R and Bosserhoff AK: Reduced expression of CYLD in human colon and hepatocellular carcinomas. Carcinogenesis 28: 21-27, 2007.

30. Song L, Liu L, Wu Z, Li Y, Ying Z, Lin C, Wu J, Hu B, Cheng SY, $\mathrm{Li}$ M, et al: TGF- $\beta$ induces miR-182 to sustain NF- $\kappa \mathrm{B}$ activation in glioma subsets. J Clin Invest 122: 3563-3578, 2012.

31. Liang HL, Hu AP, Li SL, Xie JP, Ma QZ and Liu JY: MiR-454 prompts cell proliferation of human colorectal cancer cells by repressing CYLD expression. Asian Pac J Cancer Prev 16: 2397-2402, 2015. 